https://helda.helsinki.fi

\title{
Management of coagulation factor VIII (FVIII) inhibitors
}

\author{
Lassila, Riitta
}

2019-09

Lassila , R 2019 , ' Management of coagulation factor VIII (FVIII) inhibitors ' , Thrombosis

Research, vol. 181 , pp. S60-S61 . https://doi.org/10.1016/S0049-3848(19)30369-X

http://hdl.handle.net/10138/306453

https://doi.org/10.1016/S0049-3848(19)30369-X

publishedVersion

Downloaded from Helda, University of Helsinki institutional repository.

This is an electronic reprint of the original article.

This reprint may differ from the original in pagination and typographic detail.

Please cite the original version. 


\title{
Short Review
}

\section{Management of coagulation factor VIII (FVIII) inhibitors}

\author{
Riitta Lassilaa,* \\ ${ }^{a}$ Helsinki University Hospital, Coagulation Disorders Unit, Hematology, Helsinki, Finland
}

\section{A R T I C L E I N F O}

\section{Keywords:}

Coagulation factor VIII

FVIII

FVIII inhibitors

Acquired hemophilia

\begin{abstract}
A B S T R A C T
Acquired hemophilia is a rare but severe condition, which is unknown to medical specialties outside hematology. Sudden appearance of antibodies against coagulation FVIII is diagnosed by abnormal subcutaneous large and multiple hematomas with minor injuries only. APTT is prolonged, FVIII activity variably low and inhibitor titer varies, too. The antibodies are typically occurring in women after delivery and later in life at around 60 years of life due to immunological challenges, and in association with malignant diseases. Rapid recognition of the condition and support of hemostasis together with immunosuppressive therapy is important, since the diagnostic delay is a poor prognostic sign. Coagulation experts should be consulted to arrange the management and followup of the patients. Management of bleeds consists of so-called FVIII bypassing agents, activated prothrombin complex concentrate, aPCC, recombinant FVIIa or porcine FVIII. The immunotherapy should be continued for several weeks according to the timing of the remission. The relapse rate needs to be noted in the future under similar situations as the index event occurred. Register data collection is important to characterize this lifethreatening acquired bleeding disorder.
\end{abstract}

\section{Features of coagulation FVIII}

Factor VIII, a cofactor for coagulation serine proteases is carried by von Willebrand factor (VWF) for protection from degradation (by activated protein $\mathrm{C}$, aPC) in circulation. As VWF is the key localizer of hemostasis to the vascular injury site, the targeting of FVIII to the platelet and other relevant surfaces from blood flow, secures timely relevant engagement of coagulation activity and thrombin formation at the site of injury (Fig. 1). FVIII binds to the platelet membrane phospholipids and reaches FIX to form the tenase complex. This activates FX, which then binds to another cofactor of coagulation, that is $\mathrm{FV}$, to capture prothrombin to be converted to thrombin and moreover, fibrinogen to fibrin.

The concentration of FVIII is more than 100 -fold lower $(0.0003 \mu \mathrm{M})$ than any other coagulation factor, which causes specific implications in the quantitative differences in FVIII levels. Elevated FVIII levels, i.e. above $190 \mathrm{IU} / \mathrm{dL}$, carry a significant thrombosis risk and reflect also endothelial cell activation under various conditions, and vice versa lowered FVIII levels, below $30 \mathrm{IU} / \mathrm{dL}$ cause significant impairment of hemostasis.

\section{Hemophilia $\mathrm{A}$ is either inherited or acquired}

Inherited hemophilia A, which is due to defective FVIII gene causing deficiency of FVIII leads to a lifelong bleeding tendency needing prophylactic FVIII replacement therapy. Women are the obligate carriers as daughters of hemophilia A men. An early replacement therapy by FVIII, the foreign protein, evokes an inhibitor that is a neutralizing IgG4-type alloantibody, which suddenly eliminates the benefit of the FVIII replacement therapy. Usually this complication occurs after the twentieth exposure to FVIII, and may associate with danger signals to the immune system, typically at any larger bleeding episode.

The typical sites for bleeds in traditional hemophilia are joints and less frequently muscles. However, when a sudden bleeding phenotype develops multiple large subcutaneous hematomas occur usually after a minor challenge, raising suspicion of acquired hemophilia A (AHA). In this acute bleeding disorder neutralizing autoantibodies, alias inhibitors will ensue against FVIII, upon an immunological challenge. This rare immunological complication is affecting 1:1.5 million people, and in young females relates to the postpartum period (1:350,000 pregnancies, occurring at day 1 to several months), and in patients older than 60 years to cancer or is of unknown etiology (65\%) $[1,2]$. Among females, in addition to pregnancy-associated events, gynecological and breast and other cancer types may trigger this complication. The bleeding phenotype resembles a primary hemostatic defect in this acquired disorder, and it is relatively similar to the inhibitors, which are encountered among milder forms of hemophilia. Main bleeding symptoms include subcutaneous (80\%), muscle (45\%) and gastrointestinal bleeds (25\%), and at least $65 \%$ of them require hemostatic therapy.

\footnotetext{
* Corresponding author at: Helsinki University Hospital, Coagulation Disorders Unit, Hematology, Helsinki, Finland.

E-mail address: riitta.lassila@hus.fi (R. Lassila).
} 
A

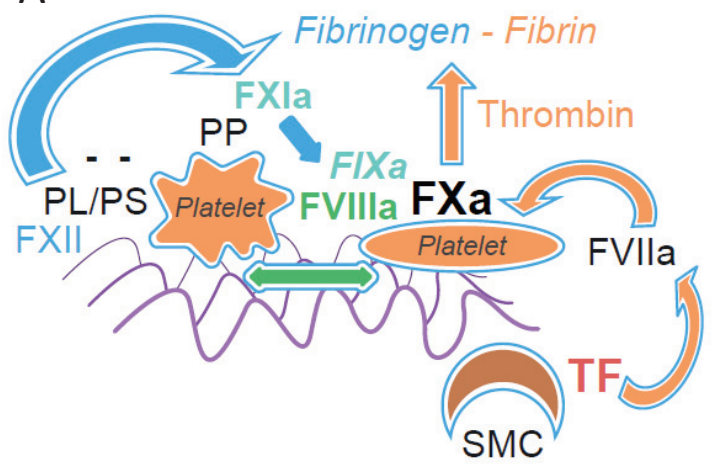

B

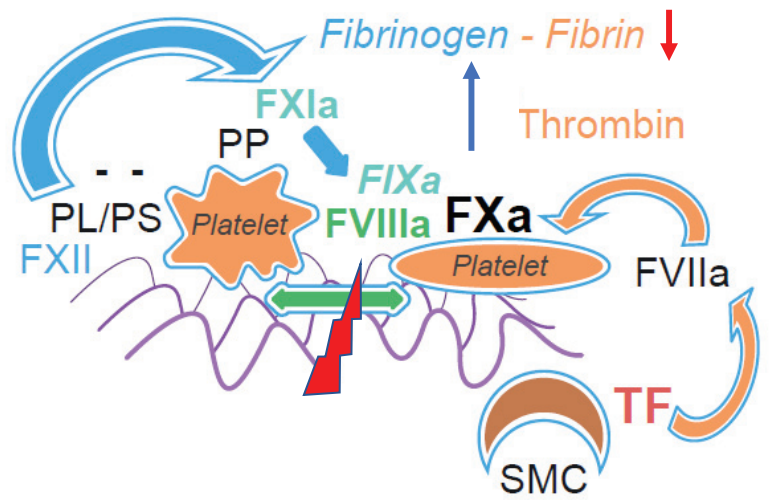

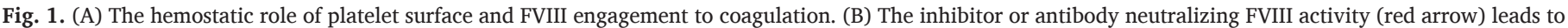

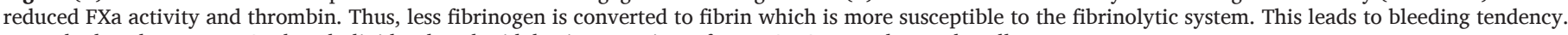
$\mathrm{PP}$, polyphosphates; PL/PS, phospholipids/phosphatidylserine; TF, tissue factor; SMC, smooth muscle cell.

\section{Diagnosis of FVIII inhibitors}

The traditional simple screening test of FVIII inhibitors is activated partial thromboplastin time, APTT. It is prolonged while PT is normal. FVIII levels are low, most often around $2-10 \mathrm{IU} / \mathrm{dL}$. The inhibitor titer varies $(10-100 \mathrm{BU} / \mathrm{mL})$, in $10 \%$ above $100 \mathrm{BU} / \mathrm{mL}$

\section{Hemostatic therapy of an inhibitor-positive patient}

Treatment targets improvement of hemostasis and eradication of the inhibitor. In life-threatening or severe cases plasmapheresis or immunoabsorption can foster the hemostatic therapy. The FVIIIbypassing remedies are needed to establish hemostasis during a bleeding event or prevention of next. They have included activated prothrombin complex concentrates (aPCC) and recombinant activated FVII (rFVIIa). Also, porcine-derived FVIII is an option.

The novel non-replacement therapies have been developed, including emicizumab, a bispecific antibody to mimic FVIII to engage FIX to the tenase complex. Thus far, its indication is exclusively congenital form of hemophilia. In addition to emicizumab, inhibitor of tissue factor pathway inhibitor, antiTFPI, concizumab and antithrombin silencing fitusiran are in the phase II-III programs to enhance thrombin generation. These three latter strategies mainly offer longterm protection against bleeds but are unlikely providing hemostatic efficacy under a significant bleed or major surgery. These hemostatic challenges will need novel approaches during such novel therapies. Some minor bleeds or surgeries can be managed with tranexamic acid (antifibrinolytic therapy) and a wait and see strategy.

\section{Overall management of patients with inhibitors against FVIII}

The bypassing therapy with either aPCC or rFVIIa provides the early management to stop bleeds as soon as possible. Several doses may be needed. The unique strength of porcine FVIII is the allowance of laboratory monitoring of FVIII.
The main management target aside reaching hemostasis is to regain tolerance to FVIII. That may be achieved in inherited hemophilia in $60-80 \%$ of the cases with immunotolerance therapy induction (ITI) with high daily doses of FVIII, usually with the therapeutic agent under use while inhibitor appeared or a VWF containing plasma concentrate. The novel extended half-life FVIII therapies which are coupled to either PEG, albumin, or immunoglobulin Fc-portion may offer ITI benefits or even reduce frequency of inhibitors, an idea which awaits to be proven.

\section{Immunosuppressive therapy}

Rapid induction of immunosuppressin is critical to control bleeding propensity and revert FVIII activity. The immunosuppressive drugs include IgG antibody production inhibiting steroids, cyclophosphamide, azathioprine and rituximab, antiCD20 inhibitor. The treatment should last until the inhibitor is below detection $(0.6 \mathrm{BU} / \mathrm{mL})$ and APTT and FVIII levels are normalized at least for a few weeks. Followup of the patient should be organized for the next 12 months and a risk profile identified, should the inhibitor appear under some future immunological challenges.

\section{Conflict of interest statement}

None declared. There is no financial support related to the manuscript or its preparation.

\section{References}

[1] P. Knoebl, P. Marco, F. Baudo, et al.; for EACH2 Registry Contributors, Demographic and clinical data in acquired hemophilia A: results from the European Acquired Haemophilia Registry (EACH2), Thromb. Haemost. 10 (2012) 622-631.

[2] L. Tengborn, F. Baudo, A. Huth-Kuhne, et al., Pregnancy-associated acquired haemophilia A: results from the European Acquired Haemophilia A (EACH2) registry, BJOG. 119 (2012) 1520-1537. 